\title{
A Hybrid Parallelization Approach for Distributed and Scalable Deep Learning.
}

\author{
S. B. Akintoye ${ }^{\mathrm{a}}$, L. Han ${ }^{\mathrm{a}, *}$, X. Zhang ${ }^{\mathrm{a}}$, H. Chen $^{\mathrm{b}}$, D. Zhang ${ }^{\mathrm{c}}$ \\ ${ }^{a}$ Department of Computing and Mathematics, Manchester Metropolitan University, UK \\ ${ }^{b}$ Department of Computer Science, University of Sheffield, UK \\ ${ }^{c}$ College of Computer Science and Technology, Nanjing University of Aeronautics and \\ Astronautics, P.R.China
}

\begin{abstract}
Recently, Deep Neural Networks (DNNs) have recorded significant success in handling medical and other complex classification tasks. However, as the sizes of a DNN model and the available dataset increase, the training process becomes more complex and computationally intensive, usually taking longer to complete. In this work, we have proposed a generic full end-to-end hybrid parallelization approach combining model and data parallelism for efficiently distributed and scalable training of DNN models. We have also proposed a Genetic Algorithm Based Heuristic Resources Allocation (GABRA) mechanism for optimal distribution of partitions on the available GPUs for computing performance optimization. We have applied our proposed approach to a real use case based on 3D Residual Attention Deep Neural Network (3D-ResAttNet) for efficient Alzheimer Disease (AD) diagnosis on multiple GPUs and compared it with the existing state-of-the-art parallel methods. The experimental evaluation shows that our proposed approach outperforms the existing parallel methods and achieves almost linear speedup with little or no differences in accuracy performance when compared with the existing non-parallel DNN models.
\end{abstract}

Keywords: Deep Learning, Genetic Algorithm, Data Parallelization, Model

Parallelization

\footnotetext{
* Corresponding author

Email address: 1.han@mmu.ac.uk (L. Han )
}

Preprint submitted to Journal of $E^{A} T_{E} X$ Templates

February 8, 2022 


\section{Introduction}

In recent time, Deep Neural Networks (DNNs) have gained popularity as an important tool for solving complex tasks ranging from image classification [1], speech recognition [2, medical diagnosis [3, 4, to the recommendation systems [5] and complex games [7, 6]. However, training a DNN model requires a large volume of data, which is both data and computational intensive, leading to increased training time.

To overcome this challenge, various parallel and distributed computing methods 8] have been proposed to scale up the DNN models to provide timely and efficient learning solutions. Broadly, it can be divided into data parallelism, model parallelism, pipeline parallelism and hybrid parallelism (a combination of data and model parallelism).

Data parallelism is a parallelization method that trains replicas of a model on individual devices using different subsets of data, known as mini-batches [9], [10]. In data parallel distributed training, each computing node or a worker contains a neural network model replica and a churn of dataset, and compute gradients which are shared with other workers and used by the parameter server to update the model parameters [11. Data parallelization is relatively easy to implement, and supported by all major frameworks such as Mxnet [12, PyTorch [13], TensorFlow [14, and etc. However, as parameters increases, the overhead for parameter synchronisation inceeases, leading to performance degradation. In addition, when a DNN model size is too big, it couldn't be executed on a single device. Hence it is not possible to perform data parallelization.

Model parallelism is a parallelization method where a large model is split, running concurrent operations across multiple devices with the same mini-batch 8. It can help to speed up the DNN training either through its implementation or algorithm. In model parallelism, each node or a worker has distinct parameters and computation of layer of a model, and also updates weight of allocated model layers. There is no need for synchronization of parameters values among the workers, often communication occurs when sharing training outputs 
between workers [15]. The major challenge of this technique is how to break the model into partitions which would be allocated to the workers [16]. Moreover, using model parallelization alone does not scale well to a large number of devices [17] as it involves heavy communication between workers. Pipelining parallelism splits the DNN models training tasks into a sequence of processing stages [59]. Each stage takes the result from the previous stage as input, with results being passed downstream immediately. However, one of the significant drawbacks of pipelining parallelism is that it is limited by the slowest stages and has limited scalability.

Recently, the combination of model and data parallelization methods known as Hybrid parallelization has been explored to leverage the benefits of both methods to minimize communication overhead in the multi-device parallel training of DNN models [18]. Song et al. [19] proposed HyPar, a hybrid parallelism architecture for DNN training with an array of DNN accelerators, and has shown through experimental evaluation that HyPar outperformed either model parallelization or data parallelization alone. Furthermore, Ono et al. [15] proposed a hybrid data-model parallel approach for sequence-to-sequence (Seq2Seq) recurrent neural network (RNN) machine translation, where a model parallelization approach was applied to the RNN encoder-decoder part of the Seq2Seq model and a data-parallel approach was applied to the attention-softmax part of the model.

Despite the performance of the existing parallelization methods, they are still subject to further improvement by optimally allocating the model computations and data partitions to the available devices for better model training performance. In this paper, we have proposed a generic hybrid parallelization approach for parallel training of DNN in multiple Graphics Processing Units (GPUs) computing environments, which combines both model and data parallelization methods. Our major contributions are as follows:

- Development of a generic full end-to-end hybrid parallelization approach for the multi-GPU distributed training of a DNN model. 
- Model parallelization by splitting a DNN model into independent partitions, formulating the network partitions-to-GPUs allocation problem as a 0-1 multiple knapsack model, and proposing a Genetic Algorithm based heuristic resources allocation (GABRA) approach as an efficient solution to optimize the resources allocation.

- Exploitation of data parallelization based on the All-reduced method and synchronous stochastic gradient descent across multiple GPUs for further acceleration of the overall training speed.

- Evaluation of the proposed approach through a real use case study - by parallel and distributed training of a 3D Residual Attention Deep Neural Network (3D-ResAttNet) for efficient Alzheimer's disease diagnosis.

The remainder of this paper is organized as follows: Section 2 reviews the related work of the study. Section 3 discusses the details of the proposed approach. In Section 4 , the experimental evaluation is described. Section 5 concludes the work.

\section{Related Work}

This section provides an overview in relation to distributed training of deep neural networks and genetic algorithms for resource optimisation.

\subsection{Parallel and Distributed Training of Deep Neural Networks (DNNs)}

As mentioned earlier, existing efforts on parallel and distributed training of DNNs can be broadly divided into three categories, which include data parallelism, model parallelism, pipeline parallelism and hybrid parallelism.

\subsubsection{Data Parallelism}

In data parallelism, a dataset is broken down into mini-batches and distributed across the multiple GPUs and each GPU contains a complete replica of the local model and computes the gradient. The gradients aggregation and updates among the GPUs are usually done either synchronously or asynchronously 


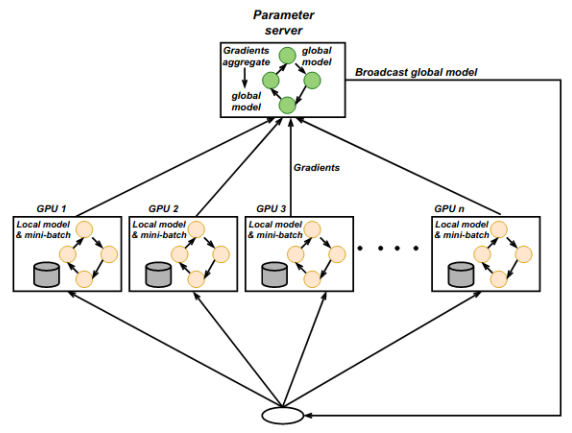

(a) Parameter server architecture

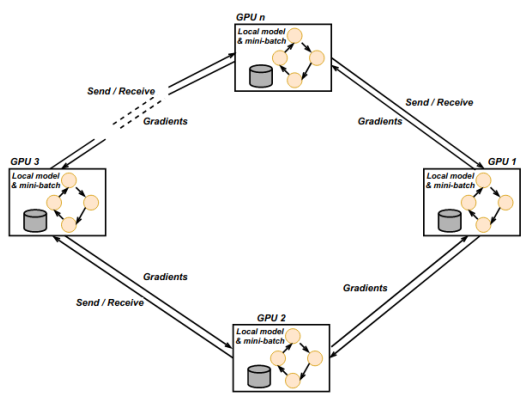

(b) AllReduce architecture

Figure 1: The conceptual data parallel training architectures

[11. In synchronous training, all GPUs wait for each other to complete the gradient computation of their local models, then aggregate computed gradients before being used to update the global model. On the other hand, in asynchronous training, the gradient from one GPU is used to update the global model without waiting for other GPUs to finish. The asynchronous training method has higher throughput in that it eliminates the waiting time incurred in the synchronous training method. In both asynchronous and synchronous training, aggregated gradients can be shared between GPUs through the two basic data-parallel training architectures: parameter server architecture and AllReduce architecture. Parameter server architecture [14] is a centralized architecture where all GPUs communicate to a dedicated GPU for gradients aggregation and updates. Figure 1aillustrates the parameter updates in data-parallel training, where each GPU computes the gradient from the local model and mini-batch. The parameter server aggregates gradients, and broadcasts the global model to all GPU for the next iteration processes. Alternately, AllReduce architecture [20] is a decentralized architecture where the GPUs share parameter updates in a ring network topology manner through the Allreduce operation as illustrated in Figure $1 \mathrm{~b}$

\subsubsection{Model Parallelism}

In model parallelization, model layers are divided into partitions and distributed across GPUs for parallel training [21, 22]. In model parallel training, 
each GPU has distinct parameters and computation of the layer of a model, and also updates weight of allocated model layers. The intermediate data such as layer outputs and gradients for the forward pass and backward pass respectively are exchanged among GPUs. Huo et al. [52 proposed a Decoupled Parallel Back-propagation (DDG), which splits the network into partitions and solves the problem of backward locking by storing delayed error gradient and intermediate activations at each partition. Similarly, Zhuang et al. 53. adopted the delayed gradients method to propose a fully decoupled training scheme (FDG). The work breaks a neural network into several modules and trains them concurrently and asynchronously on multiple devices. However, the major challenges are how to break the model layers into partitions as well as the allocation of partitions to GPUs for efficient training performance [16].

\subsubsection{Pipelining Parallelism}

Pipelining parallelism breaks the task (data and model) into a sequence of processing stages. Each stage takes the result from the previous stage as input, with results being passed downstream immediately. Various works have adopted this technique. Deep Stacking Networks (DSN) [54] overlapped neural network layer computations to optimise the performance of the training process. DSN consists of three modules, and each module computes a different hidden layer of the raw data. The raw data vector is concatenated with the output layer(s) in the lower module(s). The output of the lower module serves as input to the immediate higher module. Another deep learning system that uses the pipeline technique is PipeDream [55]. Moreover, Lee et al. [56] used the pipeline parallelism approach to overlap computation and communication for CNN training. They implement a thread in each computer server to spawn communication processes after the gradient is generated. Chen et al. [57] proposed a pipelined model parallel execution method for high GPU utilisation and used a novel weight prediction technique to achieve a robust training accuracy. 


\subsubsection{Hybrid Parallelism}

Several research works have explored both data and model parallelization methods for efficient DNN models training. Yadan et al. [23] achieved $2.2 \times$ speed-up when they trained a large deep convolutional neural network model with hybridized data and model parallelism. Krizhevsky et al. [9] used model and data parallelization techniques to train a large deep convolutional neural network and classify 1.2 million high-resolution images in the ImageNet LSVRC2010 contest into the 1000 different classes. Huang et al. 24] proposed GPipe, a scalable model-parallelism library as well as a novel batch-splitting pipelineparallelism algorithm to train large-scale convolutional and transformer-based neural networks. Shazeer et al. [25] proposed Mesh-TensorFlow where data parallelism is combined with model parallelism to improve training performance of transformer model with a huge number of parameters. In Mesh-TensorFlow, users split layers across the multi-dimensional mesh of processors and explored data parallelism technique in conjunction with the All-reduced update method. Moreover, Onoufriou et al. [26] proposed Nemesyst, a novel end-to-end hybrid parallelism deep learning-based Framework, where model partitions are trained with independent data sets simultaneously. The application of the framework on the Recurrent Neural Network (RNN) models for retail refrigeration systems, achieved cogent performance and improved the scheduling of the defrosting cycles. Similarly, Oyama et al. 27. proposed end-to-end hybrid-parallel training algorithms for large-scale 3D convolutional neural networks. The algorithms combine both data and model parallelisms to increase throughput and minimize I/O scaling bottlenecks. The results of performance evaluation with the CosmoFlow and 3D U-Net networks validate the potency of the algorithms.

The above-aforementioned approaches adopted data, model and pipeline parallelization separately or the combination of the methods to improve the performance of DNN models training. However, none of the existing methods considered the resource utilization and allocation problem in deep learning and provided solutions for efficient distributed training performance. 


\subsection{Genetic Algorithms for Resource Management Optimization}

Resource management optimization is an important research topic in distributed computing systems 28. Several works have been proposed, with different techniques for addressing resource management problems, such as scheduling [29] and allocation [30. Genetic Algorithms (GA)s are commonly used to optimize either homogenous or heterogeneous resources in distributed system environments 31 60. For instances, Gai et al. 32] proposed the Cost-Aware Heterogeneous Cloud Memory Model (CAHCM) to provide high performance cloud-based heterogeneous memory service offerings. It proposed the Dynamic Data Allocation Advanced (2DA) algorithm based on genetic programming to determine the data allocations on the cloud-based memories for the model. Mezache et al. 33. proposed a resource allocation method based on GA to minimize the number of hosts required to execute a set of cloudlet associated with the corresponding set of the virtual machine, thereby reducing excessive power consumption in the data centre. Furthermore, Jiang et al. 34] developed a mathematical model to minimize the expected total makespan and the expected total cost of the disassembly service under the uncertainty nature of the disassembly process. They proposed a multi-objective genetic algorithm based on the non-dominated sorting genetic algorithm, which shows efficiency. Mosa and Sakellariou [35] proposed a dynamic VM placement solution under consideration of heterogeneous resources. The solution used a GA to optimize the utilization of both CPU and memory with the aim to ensure better overall utilization in the cloud data centre. Devarasetty and Reddy [36] proposed an optimization method for resource allocation in the cloud with the aim to minimize the deployment cost and improve the QoS performance. They used the GA to find optimal solutions to the allocation problem. In addition to resource allocation in the cloud environment, Mata and Guardieiro [37] investigated the resource allocation in the Long-Term Evolution (LTE) uplink and proposed a scheduling algorithm based on GA to find a solution for allocating LTE resource to the user requests. Moreover, $\mathrm{Li}$ and $\mathrm{Zhu}$ [38] adopted genetic algorithm to develop a joint optimization method for offloading tasks to the mobile edge 
servers (MESs) in a mobile-edge computing environment under limited wireless transmission resources and MESs' processing resources. The outcomes of the simulation show that the proposed method can efficiently address the optimization problem of reducing the task offload completion time and resource allocation cost when compared with a single allocation offload strategy.

\section{The Proposed Approach}

In parallel and distributed computing, there are several considerations on the efficient training of DNN models including: 1) how to decompose a model or a dataset into parts/small chunks; 2) how to map and allocate these parts onto distributed resources for efficient computation as well as reducing communication overhead between computing nodes. The number of network model partitions/data partitions/mini-batches will depend on the number of network layers and the number of available GPUs in a distributed computing environment. This involves partition strategy and resource allocation.

This work has proposed a generic full end-to-end hybrid parallelization approach for efficient training of a DNN model, which combines both data and model parallelization. For data parallelization, we have exploited data parallelization based on the All-reduced method and synchronous stochastic gradient descent across multiple GPUs for acceleration of the overall network training speed. For model parallelization, model layers are partitioned individually with the aim to reduce communication overhead during the training process. We have also designed a Genetic Algorithm-based heuristic resource allocation mechanism to map and allocate partitions to appropriate resources from efficient DNN training.

Figure 2 shows the high-level architecture, including 1) model parallelization consisting of network partitions and resource allocation components; and 2) data parallelization. The details of the proposed method are presented in the following sections. The important notations in this paper are detailed in Table 1. 
Table 1: Notations

\begin{tabular}{cl}
\hline Notations & Descriptions \\
\hline$s_{i}$ & The $i-t h$ Network layer. \\
$p_{n}$ & The Network partitions of $n$ size. \\
$Q$ & Number of Network layer. \\
$d_{m}$ & The set of $m$ GPUs. \\
$t$ & Iteration. \\
$a^{t}$ & Activation at iteration $t$. \\
$w_{q}$ & The weight parameter of $q$ layer. \\
$b$ & The Batch size. \\
$l$ & The Loss function. \\
$\gamma_{t}$ & Learning rate at iteration $t$. \\
$v$ & Data point. \\
$V$ & Total data points of the dataset. \\
$b$ & Batch size. \\
$\Psi_{c}$ & The crossover operator. \\
$\Psi_{m}$ & The mutation operator. \\
$g_{s_{(i)}}^{t}$ & The gradient at partition $(i)$ at iteration $t$. \\
\hline
\end{tabular}




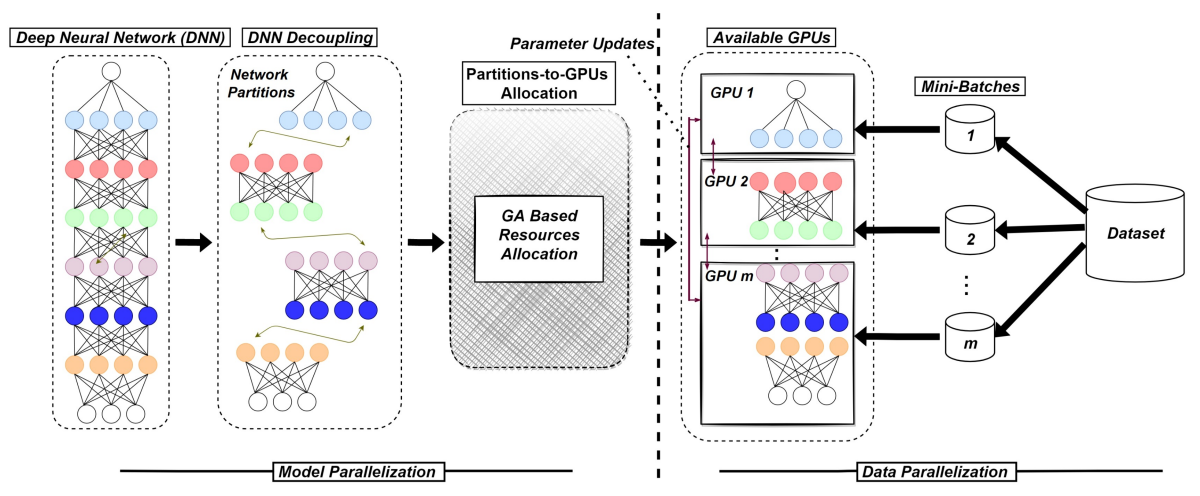

Figure 2: The high level architecture of the proposed hybrid parallelization approach

\subsection{Model Parallelization}

Model parallelization includes neural network model partitioning and Genetic Algorithm based heuristic resource allocation mechanism.

\subsubsection{Network Partitioning}

The principle of the network partitioning is based on the computation loads of each layer with the aim to reduce communication overhead during training process. The highly functional layers are partitioned individually as a single partition for even distribution of the DNN model layers. For instance, a convolution layer of CNN architecture has a large volume of weights and can be partitioned as a single partition for efficient parallel training performance.

Specifically, let's assume a model network contains a set of layers $\left\{s_{1}, \ldots, s_{q}, \ldots, s_{Q}\right\}$. The model network $P$ is split into partitions $\left\{p_{1}, p_{2}, \ldots, p_{n}\right\}$ where $p_{i}=\left\{s_{i}, s_{i}+\right.$ $\left.1, \ldots, s_{i+1}-1\right\},\left\{s_{i}, s_{i}+1, \ldots, s_{i+1}-1\right\}$ denotes a set of layers in $i$ partition such that $1 \leq i \leq n$. In addition to this, all partitions are computed simultaneously, the gradient of the partition input is passed to the next partition $(i-1)$, while the partition output is sent to partition $(i+1)$ as its new input. In forward pass, the input $a_{s_{i-1}}^{t}$ from partition $(i-1)$ is sent to partition $i$ and gives activation $a_{s_{(i+1)}-1}^{t}$ at iteration $t$. Also, In backward pass, the $g_{s_{(i+1)}-1}^{t}$ denotes the 
gradient at partition $(i+1)$ at iteration $t$. For each layer $\left(s_{i} \leq q \leq s_{i+1}-1\right)$

such that $q \leq Q$, the gradient is given as: $\hat{g}_{w_{q}}^{t}=\frac{\delta a_{s_{(i+1)}-1}^{t}}{\delta w_{q}^{t}} g_{s_{(i+1)}-1}^{t}$ which can be updated by $w_{q}^{t+1}=w_{q}^{t}-\gamma_{t} \hat{g}_{w_{q}}^{t}$ where $\gamma_{t}$ is learning rate.

$$
\hat{g}_{w_{q}}^{t-i+1}=\frac{\delta a_{s_{(i+1)}-1}^{t-i+1}}{\delta w_{q}^{t-i+1}} g_{s_{(i+1)}-1}^{t-i+1}
$$

which can be updated by:

$$
w_{q}^{t-i+2}=w_{q}^{t-i+1}-\gamma_{t-i+1} \hat{g}_{w_{q}}^{t-i+1}
$$

where $\gamma_{t-i+1}$ is learning rate.

\subsubsection{Genetic Algorithm Based Resource Allocation (GABRA)}

To enable efficient DNN model training on multiple GPUs, we have also proposed a Genetic Algorithm-based heuristic resource allocation mechanism. We formulate the problem of allocating GPUs to network partitions as a 01 multiple knapsack problem model. As previously illustrated, we consider computation load of a set of partitions $p_{i}$, where $i=\{1,2, \ldots, n\}$. We also consider the capacity of a set of available GPUs $G$, each denoted by $d_{j}$ where $j=\{1,2, \ldots, m\}$ and $d_{j} \in G$. Furthermore, we assume that each GPU runs at least one partition and each partition need to be allocated to only one GPU.

Let $C=\left(c_{i j}\right) \in \mathbb{R}^{n \times m}$ be a $n \times m$ matrix in which $c_{i j}$ is a profit of allocating GPU $i$ to partition $j$ :

$$
c_{i j}=\frac{p_{i}}{d_{j}}
$$

Also, Let $X=\left(x_{i j}\right) \in \mathbb{R}^{n \times m}$ where

$$
x_{i j}= \begin{cases}1, & \text { if gpu i is allocated to partition } \mathrm{j} \\ 0, & \text { otherwise }\end{cases}
$$


Thus, we formulate the multiple knapsack model in terms of a function $z$ as:

$$
\max _{p, x, c} z(X)=\sum_{i=1}^{n} \sum_{j=1}^{m} x_{i j} c_{i j}
$$

subject to:

$$
\begin{gathered}
\sum_{i=1}^{n} p_{i} x_{i j} \leq d_{j}, \forall j \in M=\{1,2, \ldots m\} \\
\sum_{j=1}^{m} x_{i j}=1, \forall i \in N=\{1,2, \ldots n\} \\
x_{i j}=0 \text { or } 1, \text { for } \mathrm{i}=1,2, \ldots \mathrm{n}, \text { and for } \mathrm{j}=1,2, \ldots \mathrm{m}
\end{gathered}
$$

Our goal is to find ( 8 that guarantees no GPU is overutilized and yields the maximum profit simultaneously. Thus, the objective function in equation 5 maximizes the sum of the profits of the selected partitions. The constraint Eq. 6 implies that each partition is allocated to at most one GPU, while constraints Eq. 7 ensures that the capacity of each available GPU is not exceeded.

Next, we present a Genetic Algorithm-Based Resources Allocation (GABRA) as an efficient solution to the model. Genetic Algorithm has been proven as a stochastic method to produce high-quality solutions for solving combinatorial optimization problems, particularly NP-hard problems [39, [40]. The Algorithm 2 shows the pseudo-code of the GABRA for solving GUPs-to-partitions allocation problem. It consists of four major parts: input, initialization, looping and output. In the initialization part (line 3), unlike the classical GA, the set of chromosomes which also known as initial population $\mathcal{P}(t)$ for allocating GPUs to partitions, is generated as indicated in the Algorithm 2 , by randomizing the allocation of resources without exceeding their capacities with respect to the computation load of each network partition.

The looping part contains fitness evaluation, selection, crossover and mutation functions. The objective is to optimize the total profit of allocating GPUs to partitions. The fitness evaluation validates the optimal solution condition with 


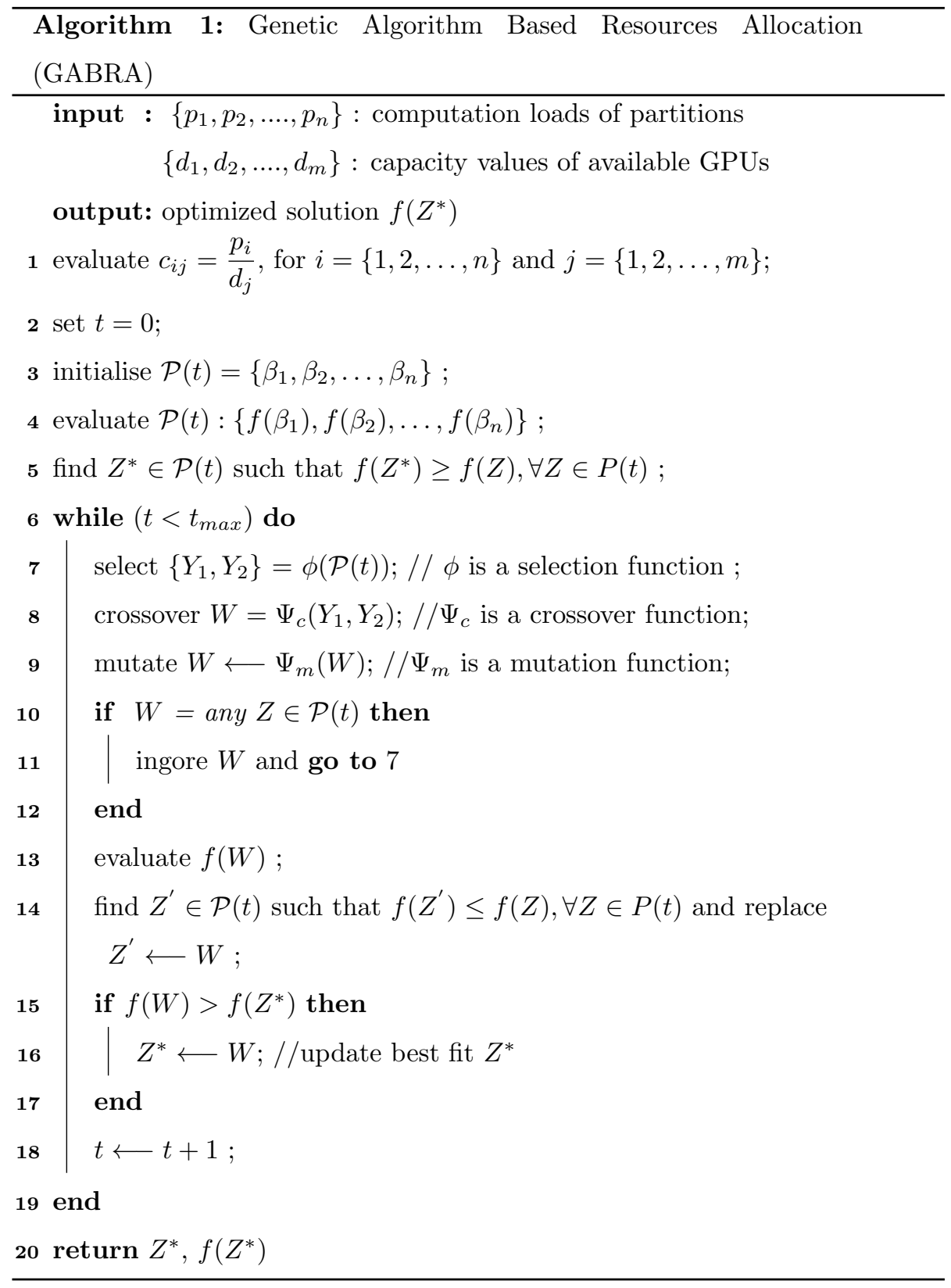




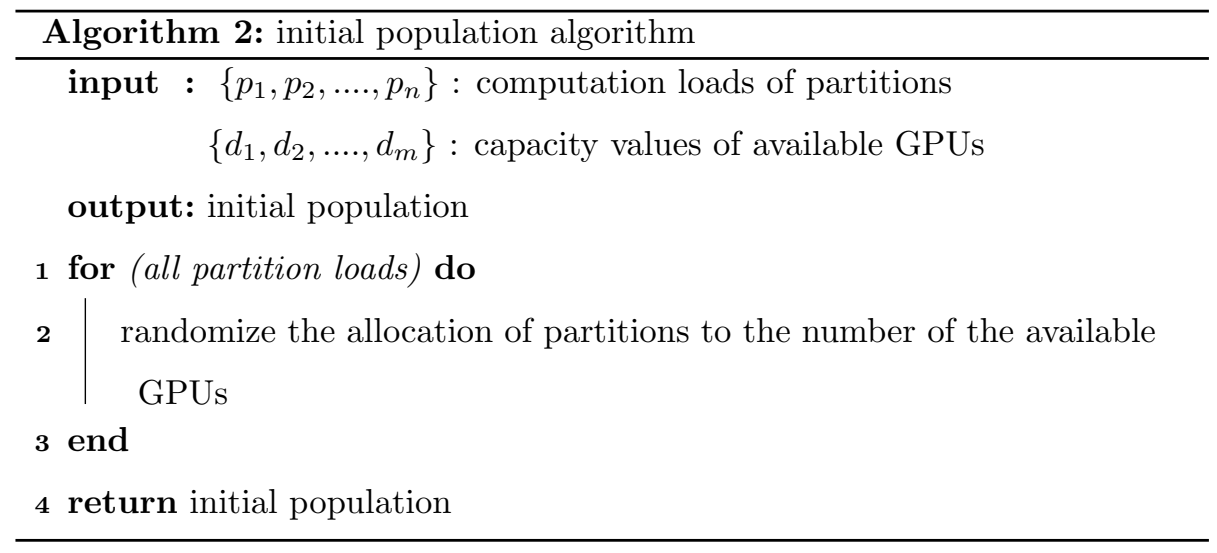

respect to the optimization objectives. Thus, the fitness value of each chromosome is calculated as:

$$
f(\beta)=\sum_{i=1}^{n} c_{i j} \beta_{i}, \text { and for } \mathrm{j}=1,2, \ldots, \mathrm{m}
$$

In the case where the optimal solution condition is not satisfying the optimization objectives, a new population is computed from an initial population of the solutions using their fitness values and genetic functions: selection, crossover and mutation functions in the looping part (lines 7- 18. We use the selection function $(\phi)$, which is based on the roulette wheel method [51] to select the best chromosomes. The selection is based on the chromosomes' fitness values, representing the total profit of allocating partitions to the available GPUs. The chromosomes with higher fitness values are selected for the generation of the next population. The midpoint crossover function $\Psi_{c}$ as described in Algorithm 3. works on two-parent chromosomes $\left\{Y_{1}, Y_{2}\right\}$ with crossover probability 0.8 and produces a new individual.

Next, the inversion mutation functions $\Psi_{m}$ is adopted where a subset of genes in a chromosome is selected and inverted to form mutated offspring. In the line 14 , the old chromosomes in the current population are replaced with the new chromosomes to form a new population. Finally, the algorithm terminates 


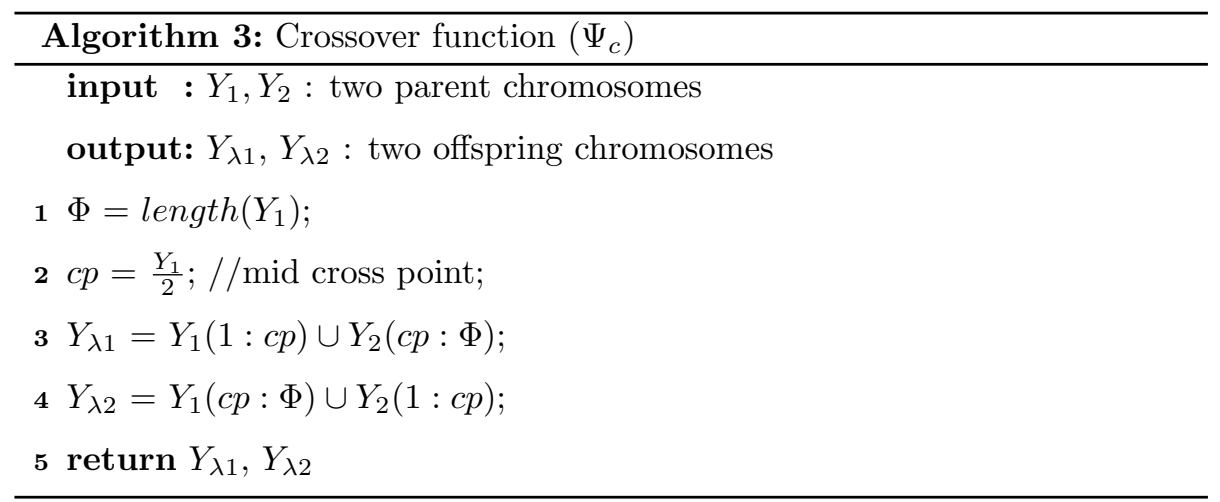

when the maximum number of generations is reached, or the optimal total profit of allocating GPUs-to-partitions is obtained.

\subsection{Data Parallelization}

To accelerate each training step, we adopt Asynchronous Stochastic Gradient Descent (ASGD) [8] as well as ring All-reduce mechanisms for parameters updates 41. ASGD achieves a faster training speed as there is no need of waiting for the slowest GPU in every iteration for the global model updates. The ring All-reduce is an optimal communication algorithm to minimize the communication overhead among the GPUs [58]. As shown in Fig. 3 all GPUs are logically arranged in a ring All-reduce topology, Each GPU sends and receives required information to update its model parameters, from the neighbour GPUs.

In all, the objective is to minimize as follows:

$$
f(w ; V)=\frac{1}{b \times m} \sum_{i=1}^{b \times m} \ell\left(w, v_{i}\right)
$$

where $f$ is a neural network, $b$ is the batch size, $m$ is the number of GPUs, $\ell$ is a loss function for each data point $v \in V$, and $w$ is the trainable parameter of the neural network.

The derivative of this objective which also referred to as the gradient is given 


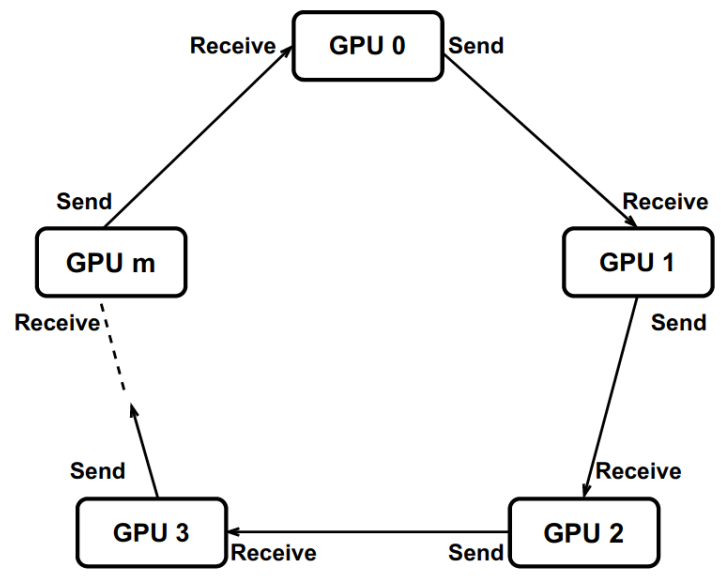

Figure 3: Ring All-reduce topology

as:

$$
\frac{\partial f(w ; V)}{\partial w}=\frac{1}{b \times m} \sum_{i=1}^{b \times m} \frac{\partial \ell\left(w, v_{i}\right)}{\partial w}
$$

In data parallellization, the gradient updates is calculated as a sum of summations, each of which is the sum of derivatives over $b$ data points, and is given as:

$$
\begin{array}{r}
\frac{\partial f(w ; V)}{\partial w}=\frac{1}{m}\left(\frac{1}{b} \sum_{i=1}^{b} \frac{\partial \ell\left(w, v_{i}\right)}{\partial w}+\frac{1}{b} \sum_{i=b+1}^{b \times 2} \frac{\partial \ell\left(w, v_{i}\right)}{\partial w}+\right) \\
\left(\ldots+\frac{1}{b} \sum_{i=b \times(m-1)+1}^{b \times m} \frac{\partial \ell\left(w, v_{i}\right)}{\partial w}\right)
\end{array}
$$

In addition, the speed of data-parallel training with $m$ GPUs can be expressed as:

$$
S T_{m}=\frac{T_{1}}{T_{m}} \times \frac{T S_{1}}{T S_{m}} \times \frac{E_{1}}{E_{m}}
$$

where $T_{1}$ is the average training time per step for using one GPU, while $T_{m}$ is the time per step for using $m$ GPUs. $E_{1}$ is the number of epochs required to converge for one GPU, while $E_{m}$ is the number of epochs required for $m$ GPUs. 


\section{Experimental Evaluation Through a Real Use Case Study}

We have applied our approach to a real case study in Neurocomputing to evaluate the effectiveness of the proposed method in this work. Previously, we have developed a 3D explainable residual self-attention convolutional neural network (3D-ResAttNet) to automatically classify discriminative atrophy localization on sMRI image for Alzheimer's Disease (AD) diagnosis [43. It is a non-parallel model and runs on a single GPU. To evaluate the proposed parallel approach, we have parallellized our previous 3D-ResAttNet model and ran it on a multiple-GPUs setting comparing the performance with and without parallelization.

Moreover, we have compared our approaches with the state-of-the-art methods including Distributed Data Parallel (DDP) and Data Parallel (DP) from PyTorch framework [44], FDG [52] and DDG [53].

\subsection{Evaluation Metrics}

We have adopted standard metrics for performance evaluation including Speedup (S), Accuracy (ACC) and Training Time (TT). The Speedup (S) is to measure the scalability and computing performance. It is defined as the ratio of the serial runtime of the best sequential algorithm for solving a problem to the time taken by the parallel algorithm to solve the same problem on multiple processors (e.g., GPUs in this case). It can be calculated as:

$$
S=T_{s} / T_{p}
$$

where $T_{s}$ represents computing time on a single machine or GPU. $T_{p}$ refers to the computing time on multiple machines or GPUs. The Accuracy (ACC) measures the classification accuracy and is defined as:

$$
A C C=(T P+T N) /(T P+T N+F P+F N)
$$

where $\mathrm{TP}=$ True positive, $\mathrm{FP}=$ False positive, $\mathrm{TN}=$ True negative and $\mathrm{FN}=$ False negative. Training Time (TT) is the time taken for training 3D- 
ResAttNet using the proposed approach and other existing distributed training methods.

\subsection{System Configuration}

We have conducted our experiments on an Amazon Web Service (AWS) EC2 P3 instance. Specifically, we used a p3.16xlarge instance consisting of 8 NVIDIA Tesla V100 GPUs developed purposely for the deep learning and Artificial intelligent crowd to provide ultra-fast GPU to GPU communication through NVLink technology. Other hardware configuration of the p3.16xlarge instance includes 128GB GPU memory, 64 vCPUs, 488GM memory, and 25Gbps network bandwidth. Additionally, software configuration /installation include: Ubuntu 18.04, Python 3.7.3, Pytorch 1.2.0, Torchvision 0.4.0, Numpy 1.15.4, Tensorboardx 1.4, Matplotlib 3.0.1, Tqdm 4.39.0, nibabel, fastai, and NVIDIA Collective Communications Library (NCCL) CUDA toolkit 10.2 - a library of multi-GPU collective communication primitives [42].

\subsection{A Use Case - Parallelization of 3D-ResAttNet For Alzheimer's Disease} (AD) Diagnosis

As described earlier, we have applied our hybrid parallelization approach to our previous non-parallel 3D-ResAttNet for automatic detection of the progression of $\mathrm{AD}$ and its Mild Cognitive Impairments (MCIs) such as Normal cohort (NC), Progressive MCL (pMCI) and Stable MCI (sMCI) from sMRI scans [43. It includes two types of classification: $\mathrm{NC}$ vs. $\mathrm{AD}$, and pMCI vs. sMCI.

\subsubsection{The High-Level Parallelization Of The System}

Fig. 4 shows the high level parallelization of our previous 3D-ResAttNet model architecture based on self-attention residual mechanism and explainable gradient-based localisation class activation mapping (Grad-CAM) to improve $\mathrm{AD}$ diagnosis performance. The 3D-ResAttNet model consists of 3D Convolutional blocks (Conv blocks), Residual self-attention block, and Explainable blocks. Conv blocks use a 3D filter for computation of the low-level feature representations. The residual self-attention block combines two important network 


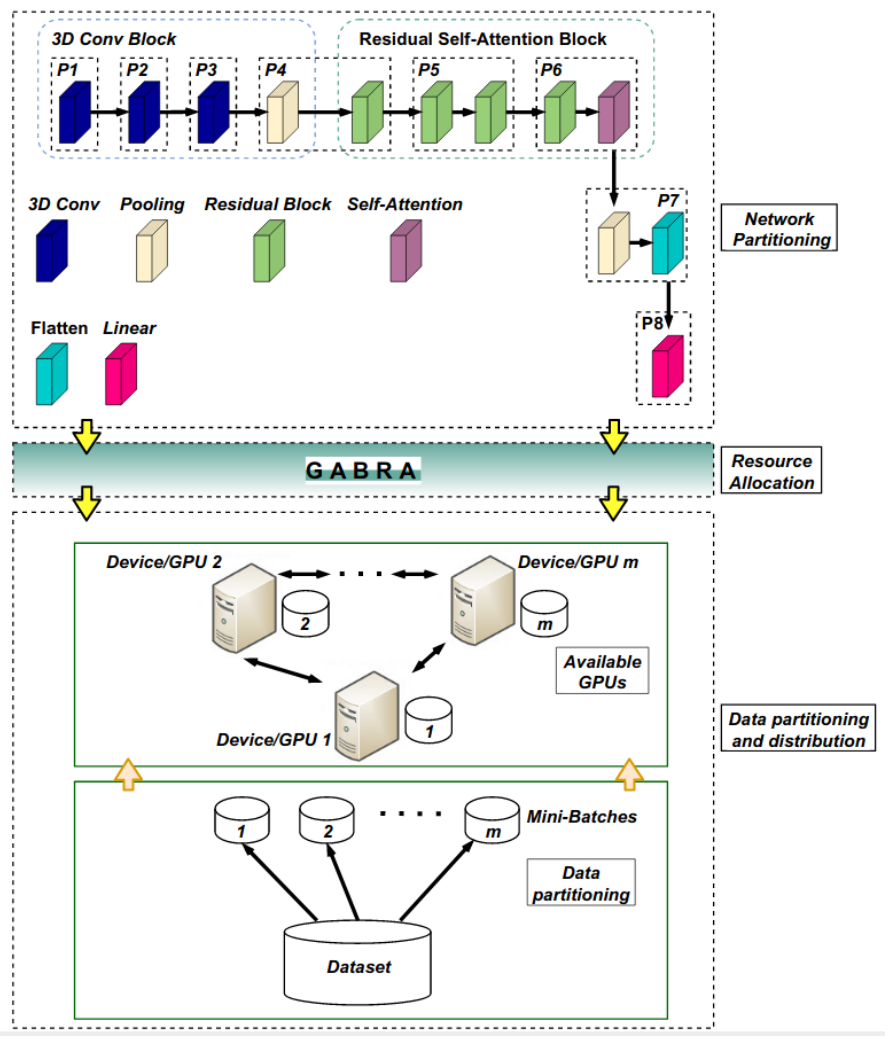

Figure 4: The hybrid parallelisation of 3D-ResAttNet

layers: Residual network layer and Self-attention layer. The residual network layer comprises two Conv blocks consisting of $3 \times 3 \times 3$ 3D convolution layers, $3 \mathrm{D}$ batch normalization and rectified-linear-unit nonlinearity layer (ReLU). The explainable block uses 3D Grad-CAM to improve the model decision.

As shown in Fig. 4, the hybrid parallelization approach for 3D-ResAttNet is divided into three phases: the splitting of 3D-ResAttNet into partitions, allocation of GPUs to partitions, and data partitioning and distribution. For data parallelization, we adopt asynchronous stochastic gradient descent as well as ring All-reduce mechanisms for parameters updates and equally distribute data parts to each GPU. For model parallelization, the network model is partitioned based on the computational complexity which usually synonymous to the number of basic operations, such as multiplications and summations, that 
each layer performs. Each Conv Block in the network consists of a $3 \times 3 \times 3$ $3 \mathrm{D}$ convolution layer, 3D batch normalization and a rectified-linear-unit nonlinearity layer (ReLU). Moreover, a convolutional layer has higher operations with complexity $\mathcal{O}\left(C_{o} \cdot C_{1} \cdot\right.$ T.H.W. $\left.K_{T} \cdot K_{H} \cdot K_{W}\right)$ where $C_{o}$ and $C_{1}$ denote the number of output and input channels respectively, $T, H$ and $W$ are image dimension, and $K_{T}, K_{H}$ and $K_{W}$ are filter dimension. Consequently, we partitioned each Conv block individually as a single partition while other layers with less computation operations are partitioned as shown in Fig. 4

Table 2: Demographic data for the subjects from ADNI database

\begin{tabular}{lcccc}
\hline Class & Number/Size & $\begin{array}{c}\text { Gender } \\
(\text { Male/Female })\end{array}$ & $\begin{array}{c}\text { Age } \\
(\text { Mean/Std })\end{array}$ & $\begin{array}{c}\text { MMSE } \\
(\text { Mean/Std })\end{array}$ \\
\hline AD & $389 / 1.4 \mathrm{~GB}$ & $202 / 187$ & $75.95 / 7.53$ & $23.28 / 2.03$ \\
pMCI & $172 / 484 \mathrm{MB}$ & $105 / 67$ & $75.57 / 7.13$ & $26.59 / 1.71$ \\
sMCI & $232 / 649 \mathrm{MB}$ & $155 / 77$ & $75.71 / 7.87$ & $27.27 / 1.78$ \\
NC & $400 / 2.4 \mathrm{~GB}$ & $202 / 198$ & $76.02 / 5.18$ & $29.10 / 1.01$ \\
\hline
\end{tabular}

\subsubsection{Dataset}

The dataset is obtained from the Alzheimer's Disease Neuroimaging Initiative (ADNI) database (http://adni.loni.usc.edu), which is the same dataset previously used for validation of our 3D-ResAttNet. The ADNI was developed in 2003 by Dr Michael W.Weiner under the public-private partnership with the aim to test the veracity of combining serial magnetic resonance imaging (MRI), positron emission tomography (PET), other biological markers, and clinical and neuropsychological assessment in measuring the progression of mild cognitive impairment (MCI) and early Alzheimer's disease (AD). The dataset contains 1193 MRI scans of four classes: 389 Alzheimer's Disease (AD), 400 Normal Cohort (NC), $232 \mathrm{sMCI}$ and $172 \mathrm{pMCI}$ patients. The demographic data for this dataset is shown in Table 2 . 
Table 3: Parallel training performance of 3D-ResAttNet using our proposed approach

\begin{tabular}{|c|c|c|c|c|c|c|c|c|}
\hline \multirow{2}{*}{ \#GPUs } & \multicolumn{4}{|c|}{ 3D-ResAttNet18 } & \multicolumn{4}{c|}{ 3D-ResAttNet34 } \\
\cline { 2 - 9 } & sMCI vs. pMCI & AD vs. NC & \multicolumn{2}{c|}{ sMCI vs. pMCI } & \multicolumn{2}{c|}{ AD vs. NC } \\
\cline { 2 - 9 } & ACC & $\begin{array}{c}\text { TT } \\
\text { (mins) }\end{array}$ & ACC & $\begin{array}{c}\text { TT } \\
\text { (mins) }\end{array}$ & ACC & $\begin{array}{c}\text { TT } \\
\text { (mins) }\end{array}$ & ACC & $\begin{array}{c}\text { TT } \\
\text { (mins) }\end{array}$ \\
\hline 1 & 0.82 & 34 & 0.95 & 62 & 0.83 & 37 & 0.96 & 68 \\
\hline 2 & 0.81 & 15 & 0.94 & 26 & 0.84 & 17 & 0.96 & 29 \\
\hline 3 & 0.81 & 13 & 0.96 & 21 & 0.84 & 14 & 0.97 & 24 \\
\hline 4 & 0.82 & 11 & 0.92 & 18 & 0.83 & 12 & 0.96 & 21 \\
\hline 5 & 0.8 & 10 & 0.94 & 17 & 0.84 & 11 & 0.97 & 19 \\
\hline 6 & 0.82 & 9 & 0.95 & 15 & 0.82 & 10 & 0.96 & 16 \\
\hline 7 & 0.81 & 7 & 0.95 & 12 & 0.84 & 8 & 0.96 & 14 \\
\hline 8 & 0.81 & 6 & 0.95 & 11 & 0.84 & 7 & 0.96 & 12 \\
\hline
\end{tabular}

\subsection{Experiments}

We have conducted experiments under different strategies:

1) We have evaluated the model performance in the parallel setting across the number of GPUs.

2) We have compared our proposed approach with four existing parallel methods including: two PyTorch generic distributed training methods: DistributedDataParallel (DDP) and DataParallel (DP), and delayed gradient parallel methods: FDG and DDG for further evaluation. DDP is a multi-process data parallel training across GPUs either on a single machine or multiple machines, while DP is for single-process multi-parallel training using multiple GPUs on a single machine [44. Both FDG and DDG were implemented by partitioning data and trained parallel models with sub-data across multiple GPUs.

In all experiments, we carried out several distributed training of 3D-ResAttNet18 and 3D-ResAttNet34 for two classification tasks: sMCI vs. pMCI and AD vs. 


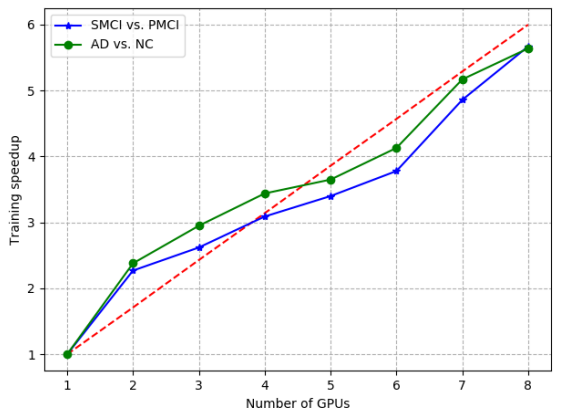

(a) sMCI vs. pMCI and AD vs. NC classification with respect to $3 \mathrm{D}-\mathrm{Res} A$ ttNet 18

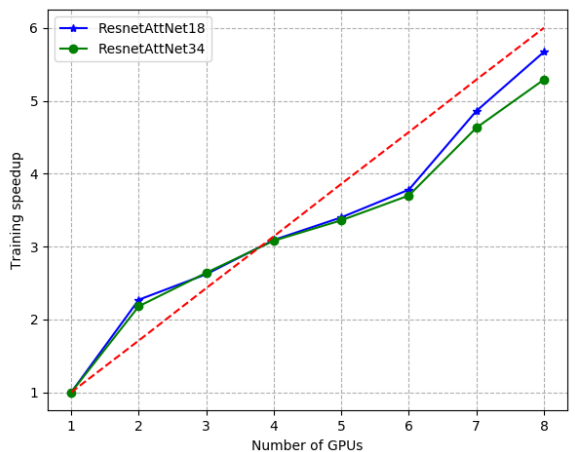

(c) 3D-ResAttNet18 vs. 3D-ResAttNet34 with respect to sMCI vs. pMCI

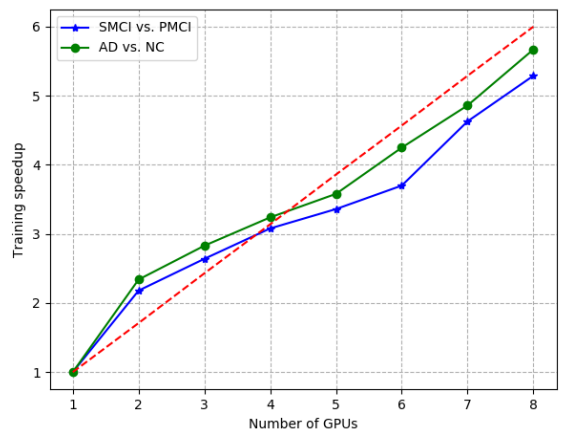

(b) sMCI vs. pMCI and AD vs. NC classification with respect to $3 \mathrm{D}-\mathrm{ResAttNet} 34$

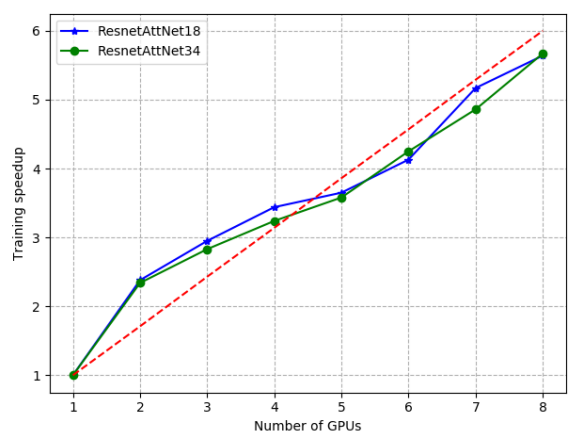

(d) 3D-ResAttNet18 vs. 3D-ResAttNet34 with respect to $\mathrm{AD}$ vs. $\mathrm{NC}$

Figure 5: Speedup of our proposed approach

NC with different number of GPUs (ranging from 1 to 8). Furthermore, we optimized model parameters with SGD, a stochastic optimization algorithm, and adopted other training parameters including a batch size of six samples, crossentropy as the loss function, and 50 epochs for better convergence. In addition, we set the initial learning rate $(\mathrm{LR})$ as $1 \times 10^{-4}$, then reduced it by $1 \times 10^{-2}$ with increased iterations.

\subsection{Experimental Results and Discussion}

\subsubsection{Performance of $3 D$-ResAttNet in the parallel setting}

We conducted the experiments on 3D-ResAttNet model for two classification tasks: sMCI vs. pMCI and AD vs. NC. Table 3 shows the experiment results 


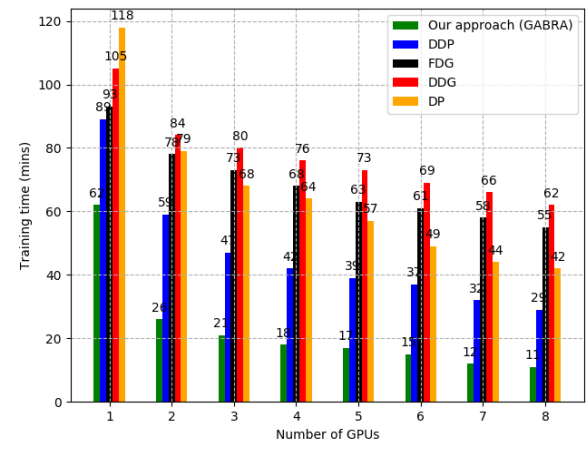

(a) Training Time of 3D-ResAttNet18 over AD vs. NC Dataset

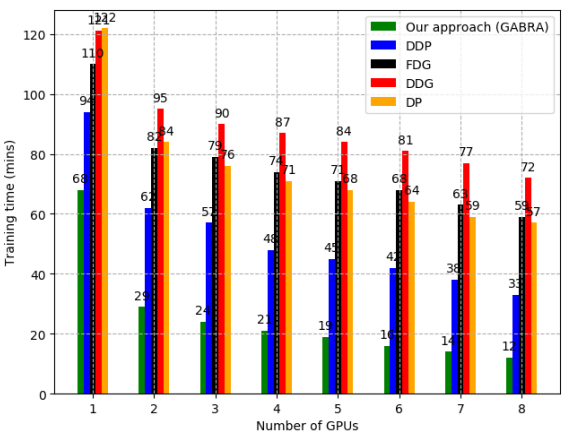

(c) Training Time of 3D-ResAttNet34 over AD vs. NC Dataset

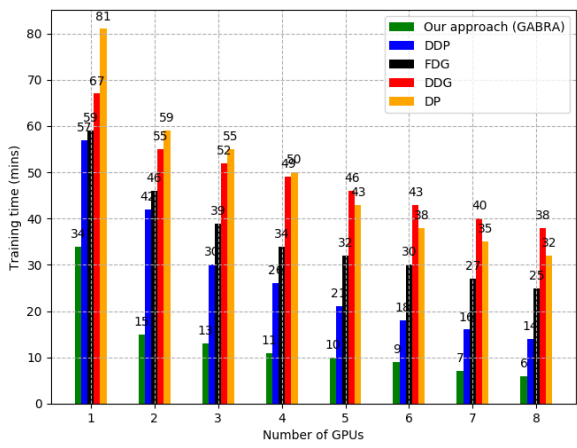

(b) Training Time of 3D-ResAttNet18 over sMCI vs. pMCI Dataset

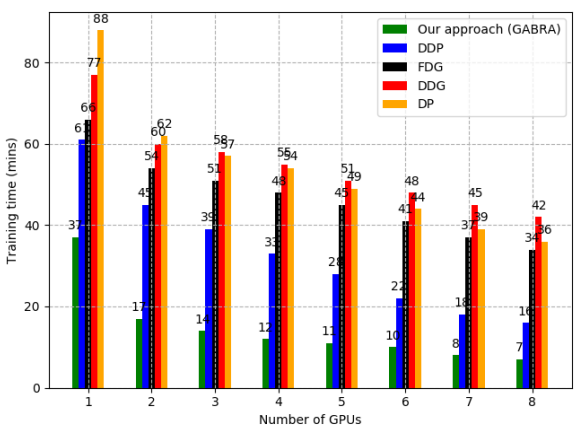

(d) Training Time of 3D-ResAttNet34 over sMCI vs. pMCI Dataset

Figure 6: Training time of proposed approach, FDG, DDG, DDP and DP

of our parallel 3D-ResAttNet (with 18 and 34 layers respectively) in terms of both training time (TT) and accuracy. Based on it, we calculated the speedup, as shown in Figs. 5a, 5b, 5c and 5d

In all cases, it is observed that our proposed approach achieves almost linear speedup, which demonstrates the scalability of our approach in that the number of GPUs is directly proportional to the training speedup performance. For instance, in $\mathrm{AD}$ vs. $\mathrm{NC}$ classification task with 3D-ResAttNet34, the training speedup for $1,23,4,5,6,7$, and 8 GPUs are $1,2.38,2.95,3.44,3.65,4.13,5.17$, and 5.64 respectively. A similar trend is also observed in the sMCI vs. pMCI classification task with 3D-ResAttNet34, the training speedup for 1, $2,3,4,5$, 
6,7 , and 8 GPUs are $1,2.27,2.62,3.09,3.40,3.78,4.86$, and 5.67 respectively.

\subsubsection{Comparison Study Between Our Proposed Approach And The Existing} Parallel Approaches (DDP, DP,DDG and FDG)

We have compared our approach with DDP, DP, DD and FDG, and the experiment results are shown in Figs. 6a, 6b, 6c, and 6d, It can be seen that our proposed approach outperforms the existing methods in terms of training time. For instance, for 3D-ResAttNet18 on AD vs. NC and sMCI vs. pMCI classification tasks, the training time incurred by our proposed approach is averagely more than $80 \%$ lower than DDP, DP, DDG and FDG. Similarly, there are related trends when comparing the proposed approach with the DDP, DP, DDG and FDG with respect to the distributed training of 3D-ResAttNet34 for two classification tasks: $\mathrm{AD}$ vs. $\mathrm{NC}$ and sMCI vs. pMCI

\subsubsection{Accuracy Comparison With The Existing Works}

Table 4 shows the accuracy comparison results from seven state-of-the-art deep neural networks and our methods. The best testing accuracies obtained in our approach are $0.97 \%$ and $0.84 \%$ for $\mathrm{AD}$ vs. NC and sMCI vs.pMCI classification respectively. The results show that our proposed approach performs efficiently when compared with the existing works in terms of accuracy. In addition, our work implements parallel distributed training of networks in a multi-GPU environment, whereas existing works are non-parallel methods.

\section{Conclusion and Future Work}

In this work, we have proposed a hybrid parallelization approach that combines both model and data parallelization for parallel training of a DNN model. The Genetic Algorithm based heuristic resources allocation mechanism (GABRA) has also been developed for optimal distribution of network partitions on the available GPUs for performance optimization. Our proposed approach has been compared with the existing state-of-the-art parallel methods and evaluated with 
Table 4: Accuracy comparison with the existing works

\begin{tabular}{lcccc}
\hline References & \multirow{2}{*}{ Model } & $\begin{array}{c}\text { Parallel } \\
\text { Training }\end{array}$ & $\begin{array}{c}\text { sMCI vs. } \\
\text { pMCI }\end{array}$ & $\begin{array}{c}\text { AD vs. } \\
\text { NC }\end{array}$ \\
\hline Hosseini-Asl et al [45] & CNN & No & N/A & 0.97 \\
Suk et al.[46] & DBM & No & 0.76 & 0.95 \\
Sarraf et al.[47] & CNN & No & N/A & 0.96 \\
Billones et al. [48] & CNN & No & N/A & 0.91 \\
Li et al.[49] & 3D CNN & No & 0.77 & 0.91 \\
Shi et al.[50] & MM-SDPN & No & 0.75 & 0.95 \\
Zhang et al.443 & 3D-ResAttNet34 & No & 0.84 & 0.97 \\
Our approach & 3D-ResAttNet34 & Yes & 0.84 & 0.97 \\
\hline
\end{tabular}

a real use case based on our previous 3D-ResAttNet model developed for efficient $\mathrm{AD}$ diagnosis. The experiment results show that the proposed approach achieves linear speedup, which demonstrates its scalability and efficient computing capability with little or no differences in accuracy performance (when compared with the existing non-parallel DNN models). Future work will be focused on further improvement of parallelization approach for efficient training performance.

\section{Acknowledgment}

The work reported in this paper has formed part of the project by Royal Society - Academy of Medical Sciences Newton Advanced Fellowship (NAF $\backslash \mathrm{R} 1 \backslash 180371)$. 


\section{References}

\section{References}

[1] Y. Li, Y. Zhang and Z. Zhu, Error-Tolerant Deep Learning for Remote Sensing Image Scene Classification, in IEEE Transactions on Cybernetics, vol. 51, no. 4, pp. 1756-1768, April 2021, doi: 10.1109/TCYB.2020.2989241.

[2] B. J. Abbaschian, D. Sierra-Sosa, A. Elmaghraby, Deep learning techniques for speech emotion recognition, from databases to models, Sensors, 2021, (4).

[3] M. Liu, J. Zhang, C. Lian and D. Shen, Weakly Supervised Deep Learning for Brain Disease Prognosis Using MRI and Incomplete Clinical Scores, in IEEE Transactions on Cybernetics, vol. 50, no. 7, pp. 3381-3392, July 2020, doi: 10.1109/TCYB.2019.2904186.

[4] M. F. J. Acosta, L. Y. C. Tovar, M. B. Garcia-Zapirain, et al., Melanoma diagnosis using deep learning techniques on dermatoscopic images, BMC Med Imaging 2021 (6).

[5] M. Schedl, Deep learning in music recommendation systems, Frontiers in Applied Mathematics and Statistics 5 (2019) 44.

[6] D. J. N. J. Soemers, V. Mella, C. Browne, O. Teytaud, Deep learning for general game playing with ludii and polygames, ArXiv, 2021, abs/2101.09562.

[7] H. Tembine, "Deep Learning Meets Game Theory: Bregman-Based Algorithms for Interactive Deep Generative Adversarial Networks," in IEEE Transactions on Cybernetics, vol. 50, no. 3, pp. 1132-1145, March 2020, doi: $10.1109 /$ TCYB.2018.2886238.

[8] J. Dean, G. S. Corrado, R. Monga, K. Chen, M. Devin, Q. V. Le, M. Z. Mao, M. Ranzato, A. Senior, P. Tucker, K. Yang, A. Y. Ng, Large scale distributed deep networks, in: Proceedings of the 25th International Conference on Neural Information Processing Systems - Volume 1, NIPS'12, 2012, pp. $1223-1231$. 
[9] A. Krizhevsky, I. Sutskever, G. E. Hinton, Imagenet classification with deep convolutional neural networks, Commun. ACM 60 (6) (2017) 84-90.

[10] J. George, P. Gurram, Distributed deep learning with event-triggered communication, ArXiv, 2019, abs/1909.05020.

[11] S. Kim, G.-I. Yu, H. Park, S. Cho, E. Jeong, H. Ha, S. Lee, J. S. Jeong,Byung-GonChun, Parallax: Sparsity-aware data parallel training of deepneural networks, in: Fourteenth EuroSys Conference 2019 (EuroSys19),Dresden, Germany., 2019, p. 15.

[12] T. Chen, M. Li, Y. Li, M. Lin, N. Wang, M. Wang, T. Xiao, B. Xu, C. Zhang, Z. Zhang, Mxnet: A flexible and efficient machine learning library for heterogeneous distributed systems, ArXiv, 2015, abs/1512.01274.

[13] PyTorch, Pytorch deep learning framework that puts python first, http://pytorch.org/, 2020 (accessed 16 Dec. 2020).

[14] M. Abadi, P. Barham, J. Chen, Z. Chen, A. Davis, J. Dean, M. Devin, S. Ghemawat, G. Irving, M. Isard, et al., Tensorflow: A system for large-scale machine learning, in: Proceedings of the 12th USENIX Conference on Operating Systems Design and Implementation, OSDI'16, USENIX Association, USA, 2016, pp. 265-283.

[15] J. Ono, M. Utiyama, E. Sumita, Hybrid data-model parallel training for sequence-to-sequence recurrent neural network machine translation, ArXiv, 2019, abs/1909.00562.

[16] R. Mayer, C. Mayer, L. Laich, The tensorflow partitioning and scheduling problem: it's the critical path!, Proceedings of the 1st Workshop on Distributed Infrastructures for Deep Learning, 2017.

[17] A. Mirhoseini, H. Pham, Q. V. Le, B. Steiner, R. Larsen, Y. Zhou, N. Kumar, M. Norouzi, S. Bengio, J. Dean, Device placement optimization with reinforcement learning, in: ICML, 2017. 
[18] M. Wang, C. Huang, J. Li, Unifying data, model and hybrid parallelism in deep learning via tensor tiling, ArXiv, 2018, abs/1805.04170.

[19] L. Song, J. Mao, Y. Zhuo, X. Qian, H. Li, Y. Chen, Hypar: Towards hybrid parallelism for deep learning accelerator array, 2019 IEEE International Symposium on High Performance Computer Architecture (HPCA), 2019.

[20] A. Sergeev, M. D. Balso, Horovod: fast and easy distributed deep learning in tensorflow, ArXiv, 2018, abs/1802.05799.

[21] Z. Jia, M. Zaharia, A. Aiken, Beyond data and model parallelism for deep neural networks, ArXiv, 2019, abs/1807.05358.

[22] A. Mirhoseini, A. Goldie, H. Pham, B. Steiner, Q. V. Le, J. Dean, A hierarchical model for device placement, in: International Conference on Learning Representations, 2018.

[23] O. Yadan, K. Adams, Y. Taigman, M. Ranzato, Multi-gpu training of convnets, CoRR, 2014, abs/1312.5853.

[24] Y. Huang, Y. Cheng, D. Chen, H. Lee, J. Ngiam, Q. V. Le, Z. Chen, Gpipe:Efficient training of giant neural networks using pipeline parallelism, ArXiv, 2019 abs/1811.06965.

[25] N. Shazeer, Y. Cheng, N. Parmar, D. Tran, A. Vaswani, P. Koanantakool, P. Hawkins, H. Lee, M. Hong, C. Young, R. Sepassi, B. A. Hechtman, Meshtensorflow: Deep learning for supercomputers, in: NeurIPS, 2018.

[26] G. Onoufriou, R. Bickerton, S. Pearson, G. Leontidis, Nemesyst: A hybrid parallelism deep learning-based framework applied for internet of things enabled food retailing refrigeration systems, Comput. Ind. 113, 2019.

[27] Y. Oyama, N. Maruyama, N. Dryden, E. McCarthy, P. Harrington, J. Balewski, S. Matsuoka, P. Nugent, B. Van Essen, The case for strong scaling in deep learning: Training large $3 \mathrm{~d}$ cnns with hybrid parallelism, IEEE Transactions on Parallel and Distributed Systems 32 (7) (2021) 1641-1652. 
[28] Z. Wesoowski, Network resource allocation in distributed systems: A global optimization framework, in: 2015 IEEE 2nd International Conference on Cybernetics (CYBCONF), 2015, pp. 267-270.

[29] A. Velarde Martinez, Scheduling in heterogeneous distributed computing systems based on internal structure of parallel tasks graphs with metaheuristics, Applied , 10 (18), 2020.

[30] L. Haji, S. Zeebaree, O. Ahmed, A. Sallow, K. Jacksi, R. Zebari, Dynamic resource allocation for distributed systems and cloud computing, Test Engineering and Management 83 (2020) 22417-2242.

[31] M. Zhang, L. Liu, S. Liu, Genetic algorithm based qos-aware service composition in multi-cloud, in: 2015 IEEE Conference on Collaboration and Internet Computing (CIC), 2015, pp. 113-118.

[32] K. Gai, M. Qiu, H. Zhao, Cost-aware multimedia data allocation for heterogeneous memory using genetic algorithm in cloud computing, IEEE Transactions on Cloud Computing (2016) 1-1.

[33] C. Mezache, O. Kazar, S. Bourekkache, A genetic algorithm for resource allocation with energy constraint in cloud computing, in: Proceedings of the International Conference on Image Processing, Production and Computer Science (ICIPCS-2016), 2016, pp. 62-69.

[34] H. Jiang, J. Yi, S. Chen, X. Zhu, A multi-objective algorithm for task scheduling and resource allocation in cloud-based disassembly, Journal of Manufacturing Systems 41 (2016) 239-255.

[35] A. Mosa, R. Sakellariou, Dynamic virtual machine placement considering cpu and memory resource requirements, in: 2019 IEEE 12th International Conference on Cloud Computing (CLOUD), 2019, pp. 196-198.

[36] P. Devarasetty, S. Reddy, Genetic algorithm for quality of service based resource allocation in cloud computing, Evolutionary Intelligence, 2019. 
[37] S. H. da Mata, P. R. Guardieiro, A genetic algorithm based approach for resource allocation in lte uplink, in: 2014 International Telecommunications Symposium (ITS), 2014, pp. 1-5.

[38] Z. Li, Q. Zhu, Genetic algorithm-based optimization of offloading and resource allocation in mobile-edge computing, Information 11 (2), 2020.

[39] T. Perry, M. Bader-El-Den, S. Cooper, Imbalanced classification using genetically optimized cost sensitive classifiers, 2015 IEEE Congress on Evolutionary Computation (CEC) (2015) 680-687.

[40] M. Bader-El-Den, M. Gaber, Garf: Towards self-optimised random forests, in: ICONIP, 2012.

[41] P. Patarasuk, X. Yuan, Bandwidth optimal all-reduce algorithms for clusters of workstations, Journal of Parallel and Distributed Computing 69 (2) (2009) 117-124.

[42] Nvidia, Nccl, https://docs.nvidia.com/deeplearning/nccl/installguide/index.html, 2021, (accessed 23 Jan. 2021).

[43] X. Zhang, L. Han, W. Zhu, L. Sun, D. Zhang, An explainable 3d residual self-attention deep neural network for joint atrophy localization and alzheimer's disease diagnosis using structural mri, ArXiv, 2020, abs/2008.04024.

[44] S. Li, Y. Zhao, R. Varma, O. Salpekar, P. Noordhuis, T. Li, A. Paszke, J. Smith, B. Vaughan, P. Damania, S. Chintala, Pytorch distributed: Experiences on accelerating data parallel training, Proc. VLDB Endow. 13 (2020) 3005-3018.

[45] . Hosseini-Asl, R. Keynton, A. El-Baz, Alzheimer's disease diagnostics by adaptation of 3d convolutional network, 2016 IEEE International Conference on Image Processing (ICIP) (2016) 126-130. 
[46] H. Suk, D. Shen, Deep learning-based feature representation for ad/mci classification, Medical image computing and computer-assisted intervention : MICCAI ... International Conference on Medical Image Computing and Computer-Assisted Intervention 16 Pt 2 (2013) 583-90.

[47] S. Sarraf, G. Tofighi, Classification of alzheimer's disease using fmri data and deep learning convolutional neural networks, ArXiv, 2016, abs/1603.08631.

[48] C. D. Billones, O. J. L. D. Demetria, D. E. D. Hostallero, P. C. Naval, Demnet: A convolutional neural network for the detection of alzheimer's disease and mild cognitive impairment, in: 2016 IEEE Region 10 Conference (TENCON), 2016, pp. 3724-3727.

[49] H. Li, M. Habes, Y. Fan, Deep ordinal ranking for multi-category diagnosis of alzheimer's disease using hippocampal mri data, ArXiv, 2017, abs/1709.01599.

[50] J. Shi, X. Zheng, Y. Li, Q. Zhang, S. Ying, Multimodal neuroimaging feature learning with multimodal stacked deep polynomial networks for diagnosis of alzheimer's disease, IEEE Journal of Biomedical and Health Informatics PP (2017) 1-1.

[51] Fengrui Yu and Xueliang Fu and Honghui Li and Gaifang Dong, Improved Fitness Proportionate Selection-Based Genetic Algorithm, Proceedings of the 2016 3rd International Conference on Mechatronics and Information Technology, 2016, pp. 136-140.

[52] Z. Huo and B. Gu and Q. Yang and H. Huang. Decoupled parallel backpropagation with convergence guarantee. In ICML, 2018.

[53] H. Zhuang and Y. Wang and Q. Liu and Z. Lin. Fully decoupled neural network learning using delayed gradients. IEEE transactions on neural networks and learning systems, PP, 2021. 
[54] L. Deng and D. Yu and J. Platt. Scalable stacking and learning for building deep architectures. In 2012 IEEE International Conference on Acoustics, Speech and Signal Processing (ICASSP), pages 2133-2136, March 2012.

[55] D. Narayanan and A. Harlap and A. Phanishayee and V. Seshadri and N. R. Devanur and G. R. Ganger and P. B. Gibbons and M. Zaharia. Pipedream: Generalized pipeline parallelism for dnn training. In Proceedings of the 27th ACM Symposium on Operating Systems Principles, pages 1-15, New York, NY, USA, 2019. Association for Computing Machinery.

[56] S. Lee and D. Jha and A. Agrawal and A. Choudhary and and W. Liao. Parallel deep convolutional neural network training by exploiting the overlapping of computation and communication. In 2017 IEEE 24th International Conference on High Performance Computing (HiPC), pages 183-192, Jaipur, 2017.

[57] C.-C. Chen and C.-L. Yang and H.-Y. Cheng. Efficient and robust parallel dnn training through model parallelism on multi-gpu platform. ArXiv, abs/1809.02839, 2018.

[58] Pitch Patarasuk and Xin Yuan. Bandwidth optimal all-reduce algorithms for clusters of workstations. J. Parallel Distrib. Comput. 69, 2 (February, 2009), 117-124. DOI:https://doi.org/10.1016/j.jpdc.2008.09.002

[59] C Kim and H Lee and M Jeong and W Baek and B Yoon and I Kim and S Lim and S Kim. torchgpipe: On-the-fly pipeline parallelism for training giant models. arXiv preprint arXiv:2004.09910. 2020.

[60] S. Li, Z. Huang and L. Han. A Genetic Algorithm Enhanced Automatic Data Flow Management Solution for Facilitating Data Intensive Applications in the Cloud. Concurrency and Computation: Practice and Experience, 2018, https://doi.org/10.1002/cpe.4844 